\title{
Gravitational light bending prevents gamma-gamma absorption in gravitational lenses
}

\author{
Hannes Thiersen* \\ NWU, South Africa \\ E-mail: 26559226@nwu.ac.za \\ Markus Böttcher \\ NWU, South Africa \\ E-mail: Markus.Bottcher@nwu.ac.za
}

The magnification effect due to gravitational lensing enhances the chances of detecting moderateredshift $(z \sim 1)$ sources in very-high energy (VHE; $E>100 \mathrm{GeV}) \gamma$-rays by ground-based Atmospheric Cherenkov Telescope facilities. It has been shown in previous work that this prospect is not hampered by potential $\gamma-\gamma$ absorption effects by the intervening (lensing) galaxy, nor by any individual star within the intervening galaxy. In this paper, we expand this study to simulate the light bending effect of a realistic ensemble of stars. We first demonstrate that, for realistic parameters of the galaxy's star field, it is extremely unlikely (probability $\leq 10^{-6}$ ) that the direct line of sight between the $\gamma$-ray source and the observer passes by any star in the field close enough to be subject to significant $\gamma \gamma$ absorption. Our simulations then focus on the rare cases where $\gamma \gamma$ absorption by (at least) one individual star might be non-negligible. We show that gravitational light bending will have the effect of avoiding the $\gamma-\gamma$ absorption spheres around massive stars in the intervening galaxy. This re-inforces prospects of using VHE $\gamma$-ray observations of lensed blazars to probe the location of the $\gamma$-ray emission region in those blazars.

4th Annual Conference on High Energy Astrophysics in Southern Africa

25-27 August, 2016

South African Astronomical Observatory (SAAO), Cape Town, South Africa

*Speaker. 


\section{Introduction}

Very-high energy (VHE; $E>100 \mathrm{GeV}$ ) $\gamma$-rays are subject to $\gamma-\gamma$ absorption from extragalactic background light (EBL) due to electron-positron pair production. The $\gamma-\gamma$ absorption effect is further intensified by the dense infrared-optical-UV radiation fields from various celestial objects. The distance at which VHE $\gamma$-rays can be detected from sources such as blazars is thus greatly reduced compared to lower energy radiation. As such, VHE $\gamma$-ray sources at large cosmological distances are required to be exceptionally bright. However, the magnification effect from gravitational lensing by intervening galaxies may extend the VHE $\gamma$-ray visibility of lensed sources to signigicantly larger distances. The most distant source of VHE $\gamma$-rays to date is the gravitationally-lensed blazar S3 $0218+357$ [3] at redshift $z=0.944$.

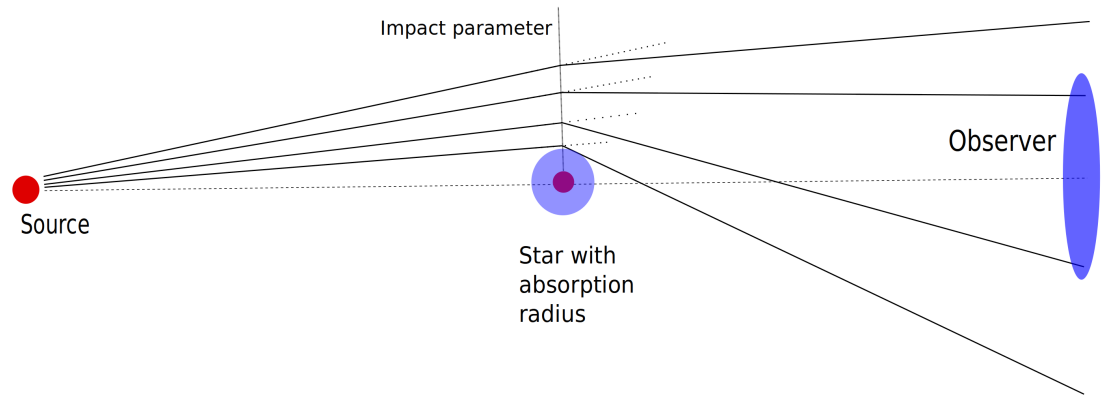

Figure 1: Schematic representation of $\gamma$-rays (black solid lines) being lensed around a star. The absorption radius of the star as blue shaded circle.

In the context of a potential VHE detection of a gravitationally-lensed blazar, it is critical to understand whether the additional infrared-optical-UV radiation fields from a lensing galaxy contribute significantly to $\gamma-\gamma$ absorption of VHE $\gamma$-rays which could effectively nullify the brightness enhancements from gravitational lensing. [1] showed that there is no significant contribution to $\gamma-\gamma$ absorption from an individual intervening star or the collective radiation field of the entire galaxy. Due to the gravitational light bending, $\gamma$-rays will pass these objects at distances much larger than $r_{\gamma \gamma}{ }^{1}$.

Figure 2 illustrates that the $\gamma$ - $\gamma$ opacity is negligible even if $\gamma$-rays pass through a galaxy. As when considering the effet of an entire galaxy, the point source approximation is no longer valid, the galaxy has been represented as a continuous disc with a de Vaucouleur brightness profile in [1]. This neglects the lensing and potential $\gamma-\gamma$ absorption effects of individual stars within the galaxy, which could possibly greatly exceed the effects found with a continuous approximation of the galaxy's gravitational and radiation field.

We therefore here extend the study of possible $\gamma-\gamma$ absorption effects in gravitational lenses when $\gamma$-rays pass through an intervening galaxy, taking into account the contribution from a realistic ensemble of individual stars within the galaxy. This is done by simulating a representative collection of stars in a galaxy and tracing $\gamma$-ray paths passing through it. We then evaluate the point

\footnotetext{
${ }^{1}$ the radial distance at which the $\gamma-\gamma$ opacity becomes significant; $\tau_{\gamma \gamma}>1$.
} 

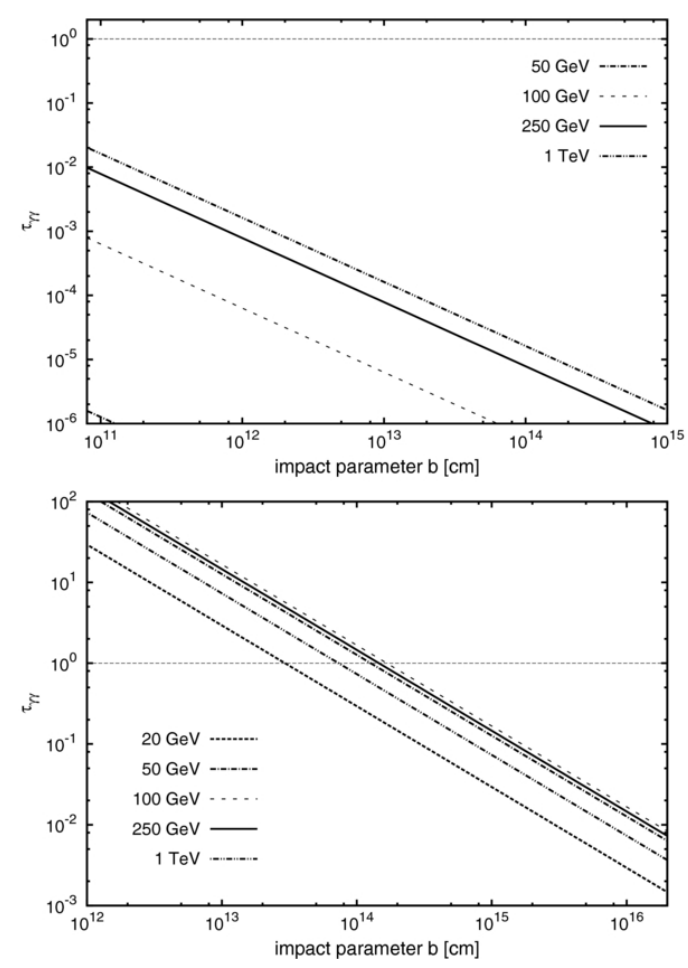

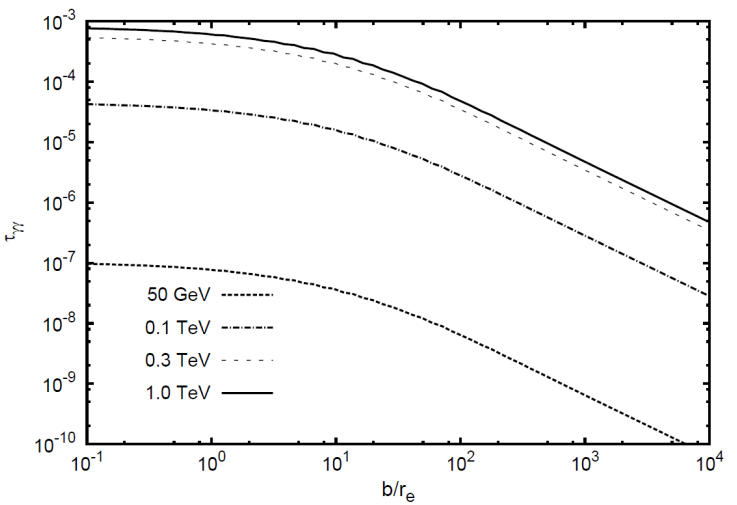

Figure 2: Left: Due to gravitiational light beniding, $\gamma$-rays will pass these stars at charactersistic minimum distances of $10^{16}-10^{17} \mathrm{~cm}$, i.e., far outside the stars' $\gamma-\gamma$ absorption radii.

Right: $\gamma-\gamma$ opacity as function of impact parameter of $\gamma$-rays from Milky-Way like galaxy with effective radius $r_{e}=0.7 \mathrm{pc}$.

[1]

of closest approach of the $\gamma$-rays to each star and compare it to the radii of $\gamma-\gamma$ absorption spheres within which significant absorption occurs.

A short summary of the numerical scheme and results are presented in the following sections. Details are described in [2].

\section{Numerical Setup}

The geometrical setup is identical to the schematic in Figure 3. The source, intervening galaxy and observer are collinearly aligned. The disc of the galaxy is seen face-on by the observer. Both the source and the observer are assumed to be a distance of $3 \mathrm{Gpc}$ from the lensing galaxy.

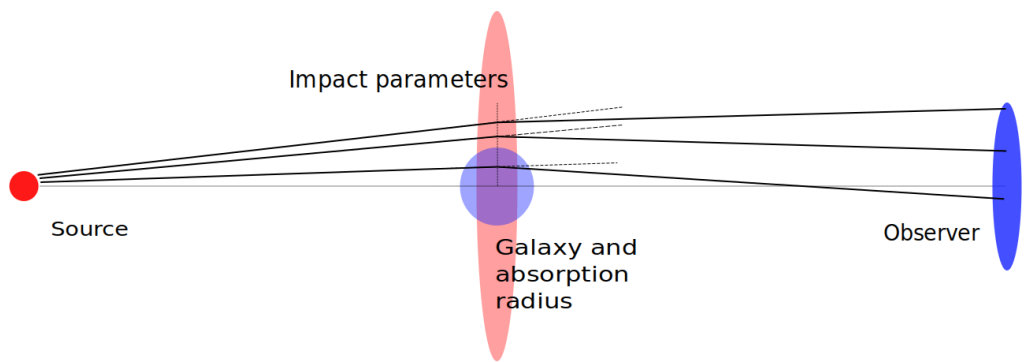

Figure 3: Schematic representation of $\gamma$-rays passing through a galaxy (red shaded disc). The absorption radius of a galaxy (blue shaded circle) as calculated with point source approximation is typically smaller than the galaxy itself.

Large lensing deflections will result in $\gamma$-rays missing the observer which render the $\gamma$-ray path irrelevant. Such deflections are highly unlikely to be realigned by deflections from other stars. 
Based on the expectation that observed $\gamma$-rays will only experience small deflections the simulation volume is restricted to a cylinder parallel to the direct line of sight with radius $r=10$ ly and height $h=1 \mathrm{kpc}$, comparable to the scale height of a typical galaxy. The expected small-angle deflections justify the approximation of the lensing effect by instantaneous deflections of the $\gamma$-ray path by a deflection angle, $\alpha$

$$
\alpha=\frac{4 G M}{c^{2} b}
$$

Where $G$ is the universal gravitational constant, $M$ the mass of the star, $c$ the speed of light and $b$ the impact parameter (distance of closest approach).

The volumetric star distribution is assumed to be uniform with an average stellar density of $n=10^{-2} \mathrm{ly}^{-3}$. The mass distribution is calculated with the Salpeter initial mass fucntion $(N(M) \propto$ $\left.M^{-2.5}\right)$.

Low mass stars $\left(M \leq 1 M_{\odot}\right)$ do not lead to significant absorption unless the $\gamma$-ray pass through the star [1]. Their deflections are also considered to be small enough to ignore. The mass range of the stars used in the simulation then spans $1 M_{\odot} \leq M \leq 100 M_{\odot}{ }^{2}{ }^{2}$ This justifies the reduction of the amount of stars in the simulation to only 250 for the specific volume.

[2] show that the probability of finding a star which could cause significant absorption on the direct line of sight is $P \lesssim 10^{-6}$. There is thus little risk for a VHE $\gamma$-ray travelling along the direct line of sight to pass close enough to a star to be absorbed. For the simulations a star is deliberately placed in the line of sight to test the unlikely worst case.

The simulation employs Monte Carlo methods to generate a star profile and then traces the path of a grid of entry positions and directions for the $\gamma$-rays through the star profile. The path impact parameters are then calculated and normalised to $r_{\gamma \gamma}$ of each individual star.

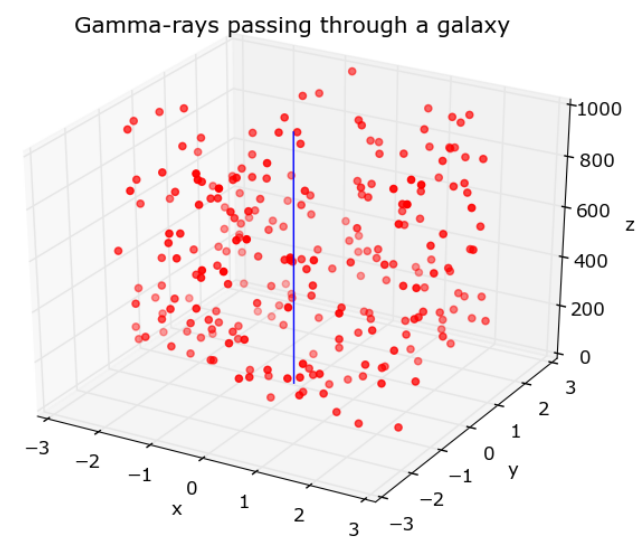

Figure 4: Visual representation of an observed $\gamma$-ray path (blue line) traced through a collection of stars (red dots). The axes are in units of parsec. [2].

Figure 4 shows the path of a single $\gamma$-ray initially travelling along the line of sight through a collection of stars as demonstration of the concept.

\footnotetext{
${ }^{2}$ The initial mass range being $0.08 M_{\odot} \leq M \leq 100 M_{\odot}$
} 


\section{Results}

Figure 5 shows the comparison between the impact parameters (normalised to $r_{\gamma \gamma}$ ) for all of the 250 stars in a representative simulation, of a lensed and unaffected observed $\gamma$-ray photon initially travelling along the direct line of sight.

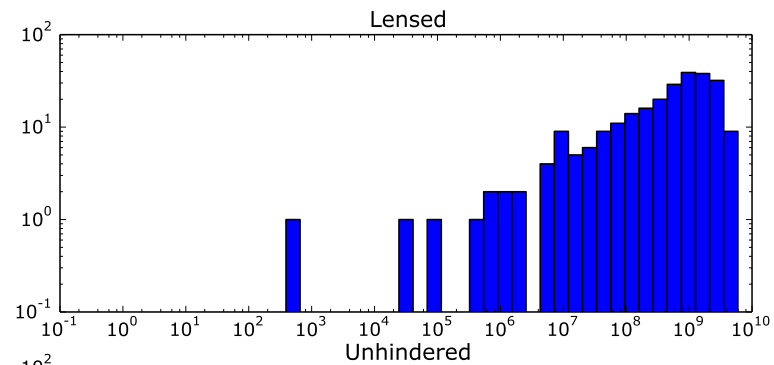

Figure 5: Histograms of results comparing the normalised impact parameters $(\mathrm{b} / \mathrm{r})$ of line of sight (red) to a lensed $\gamma$-ray (blue). Data from [2].

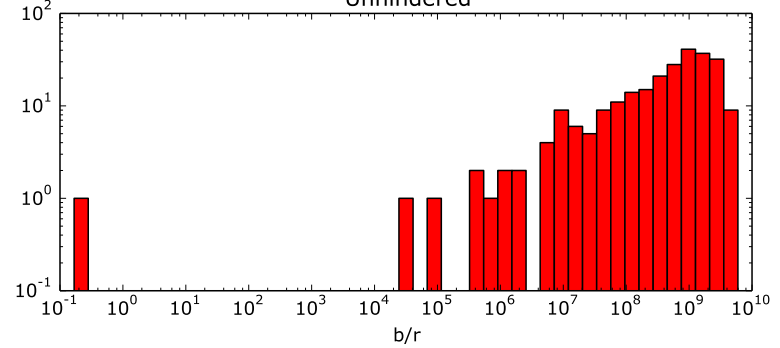

The intervening star which has been placed deliberately close to the direct line of sight, is immediately apparent in the histogram of the undeflected $\gamma$-ray. The lensed $\gamma$-ray has clearly been deflected around this deliberately placed star and avoided the region of significant $\tau_{\gamma \gamma}$. The absolute minimum normalised impact parameter of the $\gamma$-ray path is about 3 orders of magnitude larger than that of the direct line of sight.

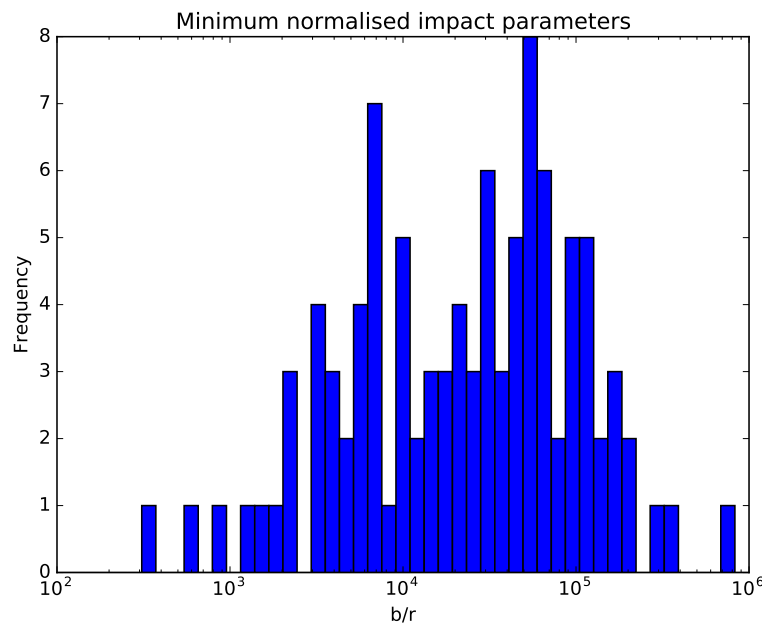

Figure 6: Histogram for the minimum impact parameters for 100 simulations. Data from [2].

In order to test the robustness of the result presented in Figure 5, we preformed 100 simulations with different random realizations of stellar distributions. Figure 6 shows a histogram of 
the absolute minimum normalised impact parameter of all the observed $\gamma$-rays of each individual simulation.

This shows that none of the observed $\gamma$-rays passed through low energy radiation fields that could cause significant absorption beyond that of the EBL. This implies that gravitational lenses do not contribute to $\gamma-\gamma$ absorption.

Globular clusters and $\mathrm{O} / \mathrm{B}$ associations were added into the simulations to improve their realism. Both cases yielded results identical to Figure 6. All these results confirm that the findings of [1] hold for a representative collection of stars in a galaxy.

There is the possibility that supermassive black holes (SMBH) in the centres of intervening galaxies or intermediate-mass black holes may significantly affect both the path and the possible $\gamma-\gamma$ absorption within the lens. Given the very small ration of SMBH to total mass, the gravitational effect of the SMBH is expected to play a significant role only within the central few $100 \mathrm{pc}$ from the SMBH. If a $\gamma$-ray passes within such a small distance of a SMBH, a posible acreation disk and/or broad-line-region radiation field (uf tge SMBH is actively accreting) may cause significant $\gamma-\gamma$ absorption. Such effects have been neglected in this study, assuming that the lensed $\gamma$-ray path passes far-enough from an active SMBH in the galactic center not to be significantly affected by it.

\section{Summary}

We investigated the claim of [1] that gravitationally lensed VHE $\gamma$-rays avoid $\gamma-\gamma$ absorption regions within the lens itself and are not subject to excessive $\gamma-\gamma$ absorption even in the case where $\gamma$-rays pass through a galaxy. By means of ray-tracing simulations, we demonstrated that this result still holds when taking into account a realistic ensemble of stars within the intervening galaxy.

This re-inforces the prospects for future VHE $\gamma$-ray dtections of gravitationally-lensed blazars at large distances.

\section{References}

[1] A. Barnacka, M. Böttcher, and Iu. Sushch. How gravitational lensing helps $\gamma$-ray photons avoid $\gamma$ - $\gamma$ absorption. ApJ, 790, 147, 2014.

[2] M. Böttcher and H. Thiersen. Gravitational light-bending prevents $\gamma \gamma$ absorption in gravitational lenses. A\&A, 595, A14, 2016.

[3] C. C. Cheung, S. Larson, J. D. Scargle, et al. Fermi Large Area Telescope detection of gravitational lens delayed $\gamma$ flares from blazar B0218+357. ApJ, 782, L14, 2014. 